\title{
Change Blindness in Proximity-Aware Mobile Interfaces
}

\author{
Michael Brock \\ Department of Engineering \\ University of Cambridge \\ Cambridge, United Kingdom \\ mob29@cam.ac.uk
}

\author{
Aaron Quigley \\ School of Computer Science \\ University of St Andrews \\ St Andrews, United Kingdom \\ aquigley@st-andrews.ac.uk
}

\author{
Per Ola Kristensson \\ Department of Engineering \\ University of Cambridge \\ Cambridge, United Kingdom \\ pok21@cam.ac.uk
}

\begin{abstract}
Interface designs on both small and large displays can encourage people to alter their physical distance to the display. Mobile devices support this form of interaction naturally, as the user can move the device closer or further away as needed. The current generation of mobile devices can employ computer vision, depth sensing and other inference methods to determine the distance between the user and the display. Once known, a system can adapt the rendering of display content accordingly and enable proximity-aware mobile interfaces. The dominant method of exploiting proximity-aware interfaces is to remove or superimpose visual information. In this paper, we investigate change blindness in such interfaces. We present the results of two studies. In our first study we show that a proximity-aware mobile interface results in significantly more change blindness errors than a non-moving interface. The absolute difference in error rates was $13.7 \%$. In our second study we show that within a proximity-aware mobile interface, gradual changes induce significantly more change blindness errors than instant changes - confirming expected change blindness behavior. Based on our results we discuss the implications of either exploiting change blindness effects or mitigating them when designing mobile proximity-aware interfaces.
\end{abstract}

\section{ACM Classification Keywords}

H.5.2. Information Interfaces and Presentation (e.g. HCI): User Interfaces-Interface design

\section{Author Keywords}

Change blindness; proxemics; proximity interaction; proximity aware interfaces; interface design

\section{INTRODUCTION}

Proximity-aware interfaces adapt as a function of the distance between the user and the display (e.g. [3, 4, 5, 8, 16]). For example, a mapping application might show an abstracted form of navigation (e.g. an arrow) at arm's length, and add progressively more details as the device is brought closer to the user (Figure 1). We conjecture that this seamless transition

\footnotetext{
Permission to make digital or hard copies of all or part of this work for personal or classroom use is granted without fee provided that copies are not made or distributed for profit or commercial advantage and that copies bear this notice and the full citation on the first page. Copyrights for components of this work owned by others than the author(s) must be honored. Abstracting with credit is permitted. To copy otherwise, or republish, to post on servers or to redistribute to lists, requires prior specific permission and/or a fee. Request permissions from permissions@ acm.org.

CHI'18, Apr 21-26, 2018, Montreal, Canada

(C) 2018 Copyright held by the owner/author(s). Publication rights licensed to ACM. ISBN 123-4567-24-567/08/06. . \$15.00
}

DOI: http: //dx.doi.org/10.475/123_4

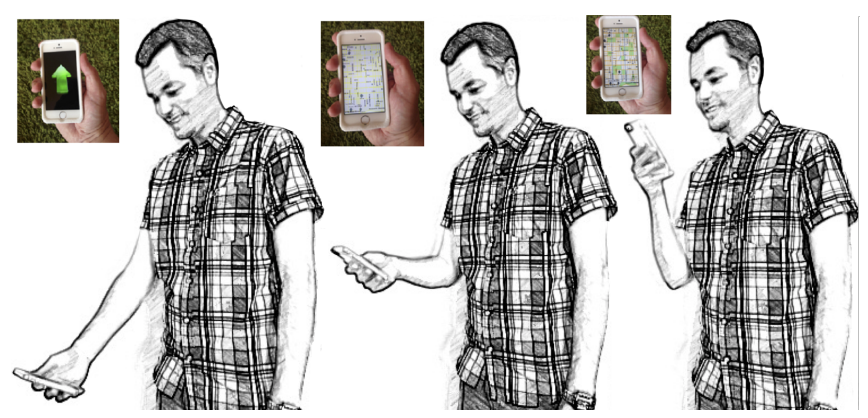

Figure 1. An illustrative example of a user interacting with a proximityaware mobile navigation application. Moving the phone closer changes the view within the application. At a far distance a simple arrow directs the user. As the user brings the phone closer, extra details are added.

from less to more detail or more to less can be improved if designers are able to exploit knowledge of when and where users experience change blindness (CB). To support such and other designs it is therefore necessary to study change blindness in the context of mobile proximity-aware interfaces.

In this paper we study how user-driven device motion of a tablet influences our ability to detect changes. With such device movements becoming more prominent due to the increase in wearable technology, it is important to not just explore the interaction space but to also understand the limitations of this interaction form in order to prevent problems. In this paper we discuss the design space that intersects proximity-aware mobile interfaces and change blindness effects and report the results of two controlled experiments that make the following contributions to the literature:

- Proximity-aware interfaces induce change blindness. We show that a proximity-aware mobile interface induces significantly more change blindness compared with a mobile interface that is held static. Participants missed on average $43 \%$ of changes when the tablet was moving while only $29 \%$ of changes were missed when the tablet was not moving.

- The effect of gradual versus instant changes, on change blindness. Prior research on change blindness has demonstrated that change detection for instant changes without a visual disruption is very high (97\%) [13]. Without visual disruptions, the act of moving a tablet resulted in $64 \%$ of gradual changes and $42 \%$ of instant changes being missed.

These findings help mobile interface designers exploit or mitigate change blindness. For example, an interface designer 
aware of change blindness effects in proximity-aware interfaces can help ensure important updates are not missed, or alternatively, change blindness can be exploited to introduce subtle invisible changes to the interface.

\section{BACKGROUND AND RELATED WORK}

Change blindness occurs when users fail to notice a single change within their field of view. During classic change blindness experiments participants are actively looking for changes and thus devote their full attention to the visual stimuli. Results in change blindness studies are consistent in that attention is required to detect changes [11], where attention is defined as "the formation of representations coherent over space and time" [10]. Change blindness implies what users do not see: what they are blind to. The opposite, change detection, emphasises what they do detect. The changes in change blindness experiments are of a single entity that does not move within the sequence shown, thus there is no actual motion of the item except for its appearance or disappearance. Therefore, a critical property of a change is that it must preserve spatio-temporal continuity [15]. A related phenomenon to $\mathrm{CB}$ is inattentional blindness (IB), the difference being that IB is entirely concerned with first-order information: "the simple presence of quantities", while in contrast CB involves second-order information: "the transitions between these quantities" [10]. An example of an IB study is Simons and Chabris" "Invisible Gorilla" [12] experiment in which participants were given the task to count ball passes between people wearing the same T-shirt colour. During the experiment a person in a gorilla costume walks through the scene. Since the participants' attention is consumed on counting ball passes, a significant number of participants failed to see the gorilla. In interfaces, subliminal cueing to regions of interest, as a means to overcome IB, has been explored with video editing experiments [7].

Klein et al. [6] suggest change blindness is due to optimisation of the perceptual system. In the real world all changes are accompanied by a certain amount of motion. If this motion exceeds a threshold it attracts attention, which as a consequence means the perceptual system will ignore minor changes and focus on the largest and dominant changes in a visual scene. If a change in the world does not trigger a diversion of attention, users cannot detect the difference in the visual scene compared to their recollection of the scene. This results in change blindness.

A simple distinction between changes is whether the change occurred instantly or gradually. Within an instant change an item is either removed or added to the scene without any transition. This induces a flicker effect at the location of the change making it easier to perceive. The flicker occurs as the change introduces a visual stimulus which is bigger than the threshold required to attract our attention. A gradual change introduces or removes an item over a longer period of time. This prevents the flicker effect as the change between any two consecutive frames does not exceed the threshold required to grab our attention.

A number of different methods have been established that can make the detection of instant changes more difficult [9]. A simple way to increase the difficulty of change detection is to obstruct the moment of change with a blank screen (gap contingent). The natural characteristics of the eye can also be exploited, for instance by carefully timing the change to occur during blinking or an eye saccade. Both of which have similar effects as a blank screen. The movement of the eye during a saccade can also be simulated by moving the entire frame at the moment of an instant change. To divert attention away from the actual change, random insertions can be made to the scene. These random insertions, known as "mud splashes", are mostly more salient than the actual change and so divert our attention away from the actual change. Lastly, changes can also be obscured by varying the camera position of a picture or film sequence. This change in perspective has the effect of changing all items by a small margin making it more difficult to pick out the actual change.

During a gradual change study trial the user is unaware of when the change will start or end. This is different to trials with instant changes with visual disruptions where the visual disruption clearly marks the moment of change. This makes the task of change detection more complicated as the participant is continuously looking for small changes as opposed to focusing on remembering visual details or scanning for large changes as is the case with instant changes. Simons reports of an experiment [13] where the overall detection rate of instant changes without visual disruptions lay at $97 \%$, the missed changes could be attributed to blinking or eye saccades. In the same study gradual changes had a detection rate of $64.3 \%$ and instant changes with visual disruptions had a detection rate of $57.4 \%$.

Studies such as by Simons and Levin [14] suggest that when users perceive a scene they do not remember individual precise details of the scene; instead they create abstract representations of the scene. This allows users to remember relevant aspects of the world. Small details in a scene that have not remembered changed be noticed.

The first major contribution of change blindness and inattentional blindness in mobile devices was by Davies et al. who demonstrated that both occur on small mobile displays [2]. Their change blindness study used an icon grid layout as is common in smartphones. The experiment used four conditions: no visual disruption, a blank screen, orientation change and a push notification pop-up. The no disruption condition saw a 93\% change detection rate whereas the lowest score was achieved with an orientation change at just $21 \%$. There was a strong correlation between the number of changes missed and the number of icons on the screen [2]. This has given rise to concerns with the addition of dynamic visual content to mobile displays, which might be missed due to change blindness [1].

In this paper, we first show that a proximity-aware mobile interface results in significantly more change blindness errors than a non-moving interface. An error occurs when a user reports no change when a change occurred, a change when no change occurred or correctly identify the item that did change. We show that within a proximity-aware mobile interface, gradual changes induce significantly more change blindness errors than instant changes. We then discuss the implications of ei- 
ther exploiting change blindness effects or mitigating them when designing mobile proximity-aware interfaces.

\section{EXPERIMENT 1}

The aim of the first experiment was to establish if mobile proximity-aware interfaces induce more change blindness than static mobile interfaces. To avoid confounds we opt for a simple design to try to maximise internal validity. The experimental design was a within-subjects experiment with one independent variable with two levels: a user not moving (NoMove) vs. moving the tablet (Move). The two conditions were counterbalanced. During the NOMOVE condition participants held the tablet at a consistent distance from them and tried to spot gradual changes, while during the MOVE condition participants had to move the tablet away from them and then back closer to them. We displayed stimulus changes as gradual changes since such changes are more difficult for participants to detect [13].

\section{Method}

\section{Participants}

We recruited 16 participants from a university campus. Their ages ranged from 21 to 33 (mean $=25.3$, sd $=3.7), 12$ were male and 4 were female. All participants had normal or corrected vision.

\section{Apparatus}

The experiment was carried out using a single mobile tablet device, an HTC Flyer tablet $(7$ inch diagonal, $1024 \times 600$ pixels). The device was connected to a Windows-7-based desktop PC using a USB cable.

The tablet's position and the participant's head position were tracked using three S250e OptiTrack cameras. The cameras were mounted to camera tripods and placed around the participant (see Figure 3 (inset)). One reflective marker was placed behind the tablet (which the user moved back and forth), while another was placed on the participant's forehead with the aid of a rubber-band, as shown in Figure 3 (inset). We ensured adequate artificial lighting was supplied from the ceiling lights to ensure a realistic environment.

To communicate between the cameras and the tablet we used OptiTrack's TrackingTools API and Google's Android Debug Bridge (ADB) module. The TrackingTools API allowed us to track individual markers and compute the distance between them. This distance was forwarded to the tablet using a TCP connection through a USB-cable between the PC and tablet.

The experiment application visualized a map interface as a sequence of $n$ pre-rendered frames. In the Move condition, the $n$ frames were mapped to the distance between the user and the tablet using linear interpolation. An individual frame $f_{d}$ for a distance $d$ was computed as:

$$
f_{d}=\left\lceil n \frac{d}{\Delta d}\right\rceil
$$

where $d \in[1,400] \mathrm{mm}$ was the distance between the user and the tablet, $\Delta d=400 \mathrm{~mm}$ was the permissible range and $n=50$ frames.

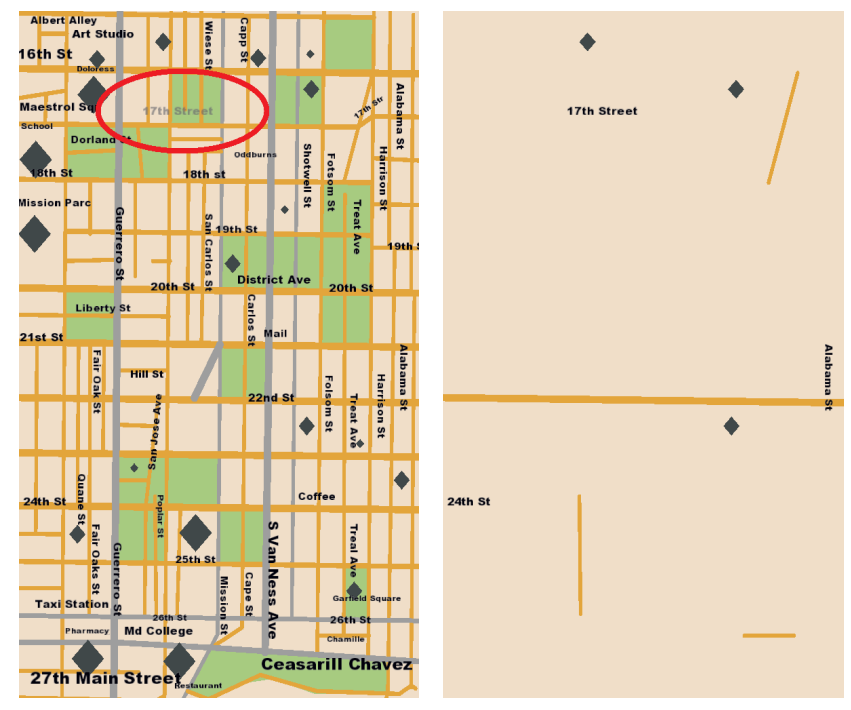

Figure 2. Left: A single gradual change in transition has been circled red. Right: All ten items used to depict a change. Each item was gradually removed from the map on the away motion of the tablet and gradually reinserted on the backwards motion. Every item was used twice, once during a NOMOVE and once during a MOVE condition making 20 gradual changes in total.

The frame number, $1-50$, monotonically increased until it reached the $50^{\text {th }}$ frame and then monotonically decreased back to the first frame. This prevented participants from forcing the visual change occurring multiple times per trial.

\section{Materials}

Participants were exposed to a map interface which displayed four different item categories: 1) terrain, 2) roads, 3) symbols and 4) labels. Ten items, which included roads, symbols and text items were used during the experiment to convey changes. Terrain changes were not included as initial testing proved these to be too easy to spot. The map was copied from a real location but individual items, such as street names were changed to ensure uniqueness.

The changes used can be seen in Figure 2. Each individual change was used once during the NOMOVE and once during the Move condition. The ten changes were distributed as follows across the item categories: four roads, three symbols and three labels. The type of change was randomised.

All frames were pre-rendered on a desktop computer and stored as individual images. For ease of use, each detail on the map had its own unique identification number, allowing predefined capture sequences to be determined and rendered. Each of these sequences consisted of 50 frames and were displayed evenly distributed across the distance moved. The images were then compiled into an Android application and installed on the tablet.

\section{Procedure}

All sessions began with two practice trials demonstrating a gradual visual change over a MOVE and NOMOVE condition. The practice trial was used to verify that participants understood the experimental setup before the actual experiment 
started. Each practice trial included one highly noticeable change that occupied most of the screen. Participants were informed that changes during the experiment were more subtle and required more focused attention.

Participants engaged in 20 trials, alternated between the MOVE and NoMove conditions. In the NoMove condition the tablet used a timing function to initiate the change after two seconds with the change itself taking $800 \mathrm{~ms}$ to complete. The $800 \mathrm{~ms}$ value was determined from a pilot study which revealed that this was the average change duration when participants were asked to perform the MOVE condition at a comfortable speed.

In the Move condition, the speed the user moved the tablet determined how quickly the change occurred. In the span of the total 50 frames per trial, the system displayed the change over ten frames starting at frame 25. During these ten frames the alpha value of an item was decreased linearly see Figure 2. At frame 35 the item was no longer visible. The change was not initiated before frame 25 to ensure the tablet was far enough to enable all participants to focus properly on the display.

When the participant moved the tablet backwards, the item which was previously removed would gradually reappear.

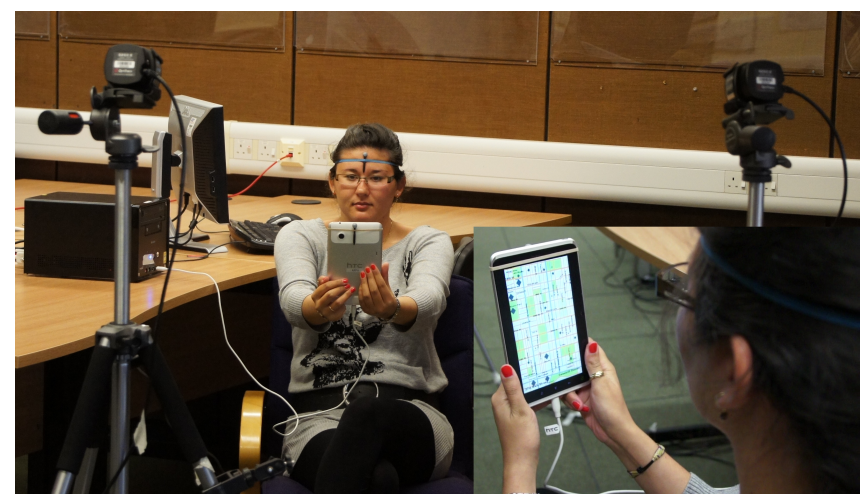

Figure 3. Participant shown with OptiTrack marker setup and engaging with the map application used in Experiment 1 and 2 (inset)

Before each trial the tablet had to be at a specific distance from the participant's head to ensure consistency across participants. The application instructed the participant to move the tablet closer or further away, as necessary.

In the NoMove condition, the tablet had to be at a distance of $35 \mathrm{~cm} \pm 2 \mathrm{~cm}$ from the participant's head for the map to be shown and a trial to begin. This equated to the distance at which a change started during a MOVE condition.

In the Move condition, the tablet had to be at a distance of $15 \mathrm{~cm} \pm 2 \mathrm{~cm}$ from the participant's head for the trial to begin. Once at this distance the trial began and the user had to move the tablet until it was $55 \mathrm{~cm}$ or more from the participant's head. At this point the tablet vibrated quickly to inform the participant that they should move the tablet back to the starting position. Once the tablet returned to the starting distance of $15 \mathrm{~cm}$ the tablet vibrated again and the participant was requested to tell the experimenter. This gave the participant two opportunities to detect each change. Participants were told that the change would occur in the middle section of the moving gesture over a prolonged distance $(8 \mathrm{~cm})$. The vibrations could also be felt during the NOMOVE condition. This was done to help participants understand when a change would occur. Participants were told that they could move the tablet at any speed they felt comfortable and could adjust the speed during and between trials. The tablet was held in the portrait position as this orientation reflects how many mobile displays are used (Figure 3).

\section{Results}

For the ten changes per condition the MOVE condition resulted in a $43 \%$ error rate (95\% confidence interval: [33\%, 52\%]) while the NOMOVE condition resulted in a $29 \%$ error rate ( $95 \%$ confidence interval: $21 \%, 38 \%$ ). A repeated-measures analysis of variance at significance level $\alpha=0.05$ showed that the difference was statistically significant $\left(F_{1,15}=5.690 ; \eta_{p}^{2}=\right.$ $0.275 ; p<0.05)$.

In other words, we have demonstrated that a proximity-aware mobile interface does induce more change blindness than a non-moving interface. In our experiment this additional induced change blindness manifested itself as a $13.7 \%$ absolute increase in error rate. This result motivates further investigation into change blindness in proximity-aware interfaces.

\section{EXPERIMENT 2}

In our second experiment we investigated the impact of the type (i.e. gradual vs. instant changes) and size of the visual change when a user is moving a tablet back and forth, again using our map application from Experiment 1 . We tested gradual versus instant changes across small, medium and large items.

With the increase in the number of conditions tested, we added a NOCHANGE condition to encourage people to state that they did not see a change if they were not sure. The order of the conditions was randomized. This was a within-subjects experiment with two independent variables, type of change with three levels: GradUAL, InStANT and NoChAnGE, and size with three levels: SMALL, MEDIUM and LARGE.

\section{Method \\ Participants}

We recruited 16 participants from a university campus. Their ages ranged from 18 to 59 (mean $=27.1, \mathrm{sd}=12.1), 7$ were male and 9 were female. Importantly, none of the participants had participated in Experiment 1. All participants had normal or corrected vision.

\section{Apparatus and Materials}

The same device setup and the same rendering process was used as in Experiment 1. Since Experiment 1 demonstrated that a proximity-aware mobile interface induced such a high increase in error rate, we decided to explore an interface that should make it slightly easier for participants to detect visual changes. We did this by using a more saturated color palette.

We prerendered three frame sequences. One sequence had 24 gradual changes (GRADUAL condition), one had 24 instant changes (INSTANT condition) and one had no changes 


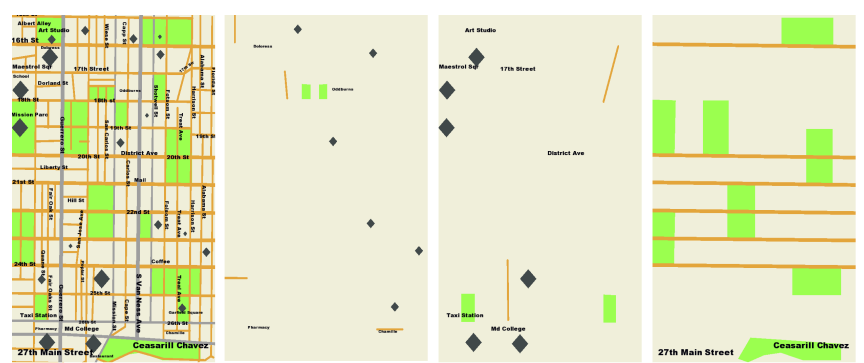

Figure 4. Full rendered map and isolated small, medium and large items which were used as changes.

(NoCHANGE condition), this would be shown 24 times. Figure 4 illustrates the map interface and small, medium and large changes.

\begin{tabular}{lllll} 
Item & Width Mean & Width SD & Height Mean & Height SD \\
\hline Symbol & 3.4 & 0.0 & 4.0 & 0.0 \\
Terrain & 3.6 & 0.1 & 6.3 & 0.0 \\
Text & 8.8 & 0.7 & 1.0 & 0.0 \\
& Length Mean & Length SD & Width Mean & Width SD \\
\hline Road & 12.0 & 1.2 & 0.9 & 0.1 \\
& \multicolumn{2}{c}{ Table 1. Small changes in millimetres }
\end{tabular}

\begin{tabular}{lllll} 
Item & Width Mean & Width SD & Height Mean & Height SD \\
\hline Symbol & 7.2 & 0.0 & 8.1 & 0.0 \\
Terrain & 5.6 & 0.2 & 12.4 & 0.1 \\
Text & 15.5 & 1.2 & 1.7 & 0.1 \\
& Length Mean & Length SD & Width Mean & Width SD \\
\hline Road & 26.8 & 0.4 & 0.9 & 0.0 \\
& \multicolumn{2}{c}{ Table 2. Medium changes in millimetres }
\end{tabular}

\begin{tabular}{lllll} 
Item & Width Mean & Width SD & Height Mean & Height SD \\
\hline Terrain & 18.0 & 11.4 & 18.7 & 5.7 \\
Text & 33.0 & 0.1 & 1.3 & 1.2 \\
& Length Mean & Length SD & Width Mean & Width SD \\
\hline Road & 90.0 & 0.0 & 1.3 & 0.0 \\
\multicolumn{2}{c}{ Table 3. Large changes in millimetres }
\end{tabular}

The 24 changes were divided equally into six changes for different item categories (roads, terrain, symbols and labels). In addition, eight of these 24 changes were classified as small, medium and large changes, as shown in Figure 4. Due to the nature of a map, symbols were only spread across small and medium changes, while the other item categories were divided across changes of all sizes.

The distinction between a small, medium and large change was defined by an estimate of the pixel area occupied by an item. Detailed measurements are given in Tables 1 to 3. The width and height for terrain, symbols and labels were measured by fitting bounding boxes around the items. Road lengths were measured as the distance from one end to the other. Road widths were measured by their thickness.

Label length and font size was varied in order to increase the width or height of a given piece of text to make it approximately equivalent in area to other items. Roughly half of the changes occurred on the top half of the display, while the other half occurred on the lower half of the display.

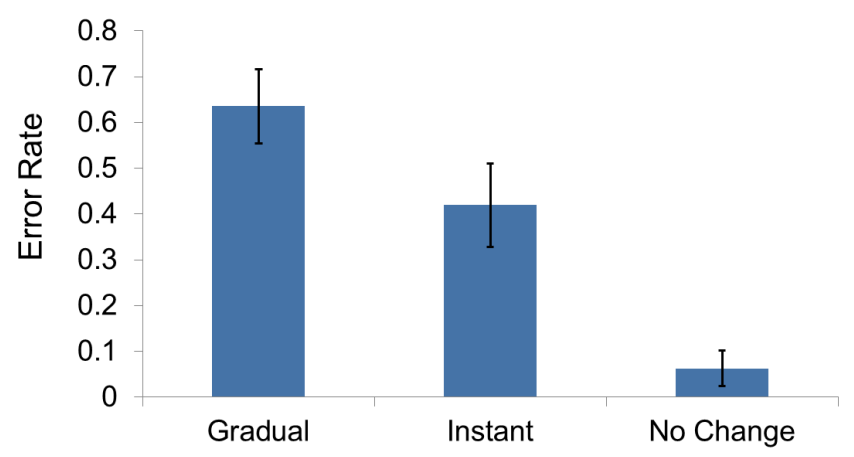

Figure 5. Mean number of errors and $95 \%$ confidence intervals in the GradUAL, InSTANT and NOChANGE conditions of Experiment 2.

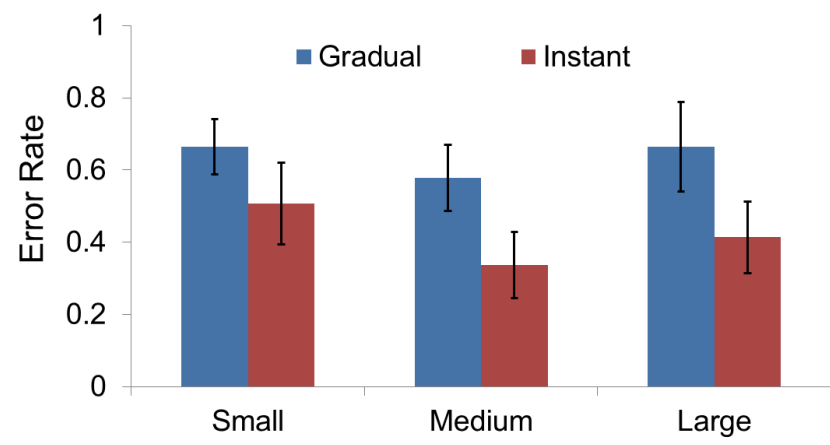

Figure 6. Mean number of errors and $95 \%$ confidence intervals across the Small, Medium and Large conditions in the Gradual vs. INSTANT conditions.

\section{Procedure}

The procedure was identical to Experiment 1 except for the following. In total, there were 72 individual trials, with 24 trials per condition (GRADUAL, INSTANT and NOCHANGE). The study was fully counterbalanced across the conditions.

An instant change occurred between two consecutive frames. The random point at which an instant change occurred was always between the minimum and the maximum distance at which a gradual change occurred.

\section{Results}

For the 24 changes per condition the GRADUAL condition resulted in a error rate of $64 \%$, the INSTANT condition resulted in a error rate of $42 \%$ and the NOCHANGE condition resulted in an error rate of $6 \%$ (see Figure 5).

In the GRADUAL condition SMALL changes resulted in a $66 \%$ error rate, MEDIUM changes resulted in a $58 \%$ error rate and LARGE changes resulted in a 66\% error rate. In the INSTANT condition SMALL changes resulted in a $51 \%$ error rate, MEDIUM changes resulted in a 33\% error rate and LARGE changes resulted in a $41 \%$ error rate (see Figure 6). Repeated measures analysis of variance at the $\alpha=0.05$ significance level revealed that the differences between the conditions were statistically significant $\left(F_{2,30}=101.896, \eta_{2}^{2}=\right.$ $0.872, p<0.0001)$. Further, Bonferroni-corrected post-hoc 
tests revealed that all pair-wise differences between the conditions were statistically significant $(p<0.001)$.

There was a high proportion of instant changes in Figure 6 that remained unnoticed in both the forwards and backwards motion of the tablet. We conjecture that the actual motion of the tablet in our studies induced change blindness as the device motion is interfering with the obvious change in the visual field.

In summary, Experiment 2 confirmed that known existing change blindness effects still holds for a mobile proximityaware interface: gradual changes are significantly more difficult to detect.

\section{DISCUSSION}

Change blindness theory predicts that gradual changes are harder to detect than instant changes without visual disruptions [10]. Our results follow the theory but the difference we measured is much greater than what would have been expected from the literature. Davies et al. [2] report of $93 \%$ overall change detection rate with instant changes without a visual disruption on a mobile screen. Using a proximity-aware interface in our second experiment, participants only achieved a $58 \%$ detection rate in our instant change condition. Within our gradual change condition only $36 \%$ of the changes were detected while Simons [13] reports a $64 \%$ detection rate. It must be stressed that participants were exposed to each change twice; once during the forwards motion and once during the backwards motion of the tablet. In contrast, in conventional $\mathrm{CB}$ studies the change is only shown once. We conjecture that since our interface gradually removed single items it created an inconsistent state which allowed users to more easily detect the change [13].

\section{Limitations}

To what extent our results are attributed to display motion as opposed to the user performing the motion themselves requires further research. This could be achieved through the use of a robotic arm that moves the display similar to how users moved the tablet in this study. The nature of the studies meant participants were actively looking for changes instead of using the map interface for navigation. The results suggests that if participants were occupied with a primary task the error rates would be higher. Two more aspects of our studies aided in change detection; firstly, our map interface introduced an inconsistent state when an item became semi-transparent. This would have allowed participants to spot a change more easily than would be possible if the item had changed colour and thus avoided an inconsistent state. Secondly, our two studies used a tablet, even though a smartphone would have been more realistic. We believe running the map application on a smaller form factor device would result in even higher error rates.

\section{Design Implications}

Participants were more prone to experience change blindness when the changes were gradual compared to when they were instant. The overall implication is that when moving a display back and forth, such as when looking at a map or any other dynamic interface on a mobile display, users can suffer from change blindness and might miss changes. These missed changes occurred for both gradual as well as instant changes. This might be missed updates to traffic information, missed routing details, or even missed location updates. While our results demonstrate a limitation for mobile application designers to consider, it is also an opportunity for them to explore. When change blindness is viewed as a limitation, designers need to consider when and how to provide visual updates to mobile displays under motion. When the aim is to exploit change blindness, careful execution of the change can ensure that a high proportion of users will not see the change. Within our experiment all participants were expecting changes and had a rough idea when changes would occur. Without this knowledge we believe the detection rate would be considerably lower. Instant changes are the norm in interfaces but as we have shown we are not immune to change blindness when the device is being moved. We therefore suggest more care is taken when considering how an interface is designed in relation to the importance of changes and device motion.

With this knowledge of change blindness we can suggest that important changes should not use gradual changes, especially if there is a chance that the device might be in motion. With the ability to sense device motion, changes can be designed to be dependent on the device's current state of motion as well as the priority of the change. Whenever the device is in motion additional precaution should be taken to ensure that these changes are not missed. Using high saliency alone is not always enough, the visual impression of the entire scene must be taken into consideration.

\section{CONCLUSIONS}

In this paper we have presented the results of two studies. First, we have shown that proximity-aware interfaces induce more change blindness errors than non-moving interfaces. Second, we replicated results from classic change blindness experiments in which it was found that gradual changes are harder to detect than instant changes. The highest error rates occurred for gradual change across small, medium and large updates. These are important findings as they relate both to mitigating and exploiting change blindness in new proximity-aware mobile interfaces. We suggest future work should not only be restricted to device motion, as in our experiment, but extended to user motion. Such a future investigation could potentially give rise to a further useful design implication: if change blindness is as prevalent in user motion as it is with device motion, then ubiquitous large displays could exploit change blindness induced due to the user's forward motion to hide irrelevant changes.

\section{ACKNOWLEDGMENTS}

P.O.K. was supported in part by a Google Faculty award and EPSRC grants EP/N010558/1 and EP/N014278/1.

\section{REFERENCES}

1. Matthias Böhmer, Christian Lander, Sven Gehring, Duncan P. Brumby, and Antonio Krüger. 2014. Interrupted by a Phone Call: Exploring Designs for Lowering the Impact of Call Notifications for Smartphone Users. In Proceedings of the SIGCHI 
Conference on Human Factors in Computing Systems (CHI '14). ACM, New York, NY, USA, 3045-3054. DOI : http://dx.doi.org/10.1145/2556288.2557066

2. Thomas Davies and Ashweeni Beeharee. 2012. The Case of the Missed Icon: Change Blindness on Mobile Devices. In Proceedings of the SIGCHI Conference on Human Factors in Computing Systems (CHI'12). ACM, New York, NY, USA, 1451-1460. DOI :

http://dx.doi.org/10.1145/2207676.2208606

3. Jakub Dostal, Per Ola Kristensson, and Aaron Quigley. 2014. Estimating and Using Absolute and Relative Viewing Distance in Interactive Systems. Pervasive and Mobile Computing 10, Part B, (Feb. 2014), 173-186. DOI : http://dx.doi.org/10.1016/j.pmcj.2012.06.009

4. Saul Greenberg, Nicolai Marquardt, Till Ballendat, Rob Diaz-Marino, and Miaosen Wang. 2011. Proxemic Interactions: The New Ubicomp? Interactions 18, 1 (Jan. 2011), 42-50. DOI : http://dx.doi.org/10.1145/1897239.1897250

5. Chris Harrison and Anind K. Dey. 2008. Lean and Zoom: Proximity-aware User Interface and Content Magnification. In Proceedings of the SIGCHI Conference on Human Factors in Computing Systems (CHI '08).

ACM, New York, NY, USA, 507-510. DOI : http://dx.doi.org/10.1145/1357054.1357135

6. Raymond Klein, Alan Kingstone, and Amanda Pontefract. 1992. Orienting of Visual Attention. In Eye Movements and Visual Cognition, Keith Rayner (Ed.). Springer New York, 46-65. DOI :

http://dx.doi.org/10.1007/978-1-4612-2852-3_4

7. Deepti Pappusetty and Hari Kalva. 2014. Effect of Subliminal Cueing on In-attentional Blindness. In Proceedings of the 1st International Workshop on Perception Inspired Video Processing (PIVP '14). ACM, New York, NY, USA, 39-40. DOI : http://dx.doi.org/10.1145/2662996.2666156

8. Thorsten Prante, Carsten Röcker, Norbert Streitz, Richard Stenzel, Carsten Magerkurth, Daniel van Alphen, and
Daniela Plewe. 2003. Hello.Wall - Beyond Ambient Displays. In Video and Adjunct Proceedings of Ubicomp'03. Springer, Seattle, WA, USA, 277-278.

9. Ronald A. Rensink. 2002. Change Detection. Annual Review of Psychology 53, 1 (2002), 245-277. D0I : http: //dx.doi.org/10.1146/annurev.psych.53.100901.135125

10. Ronald A. Rensink. 2009. Attention: Change Blindness and Inattentional Blindness. In Encyclopedia of Consciousness. Number v. 1. Elsevier Science, 47-59. DOI :

http://dx.doi.org/10.1016/B978-012373873-8.00006-2

11. Ronald A. Rensink, J. Kevin O'Regan, and James J. Clark. 1997. To See or Not to See: The Need for Attention to Perceive Changes in Scenes. Psychological Science 8 (1997), 368-373. DOI : http://dx.doi.org/10.1111/j.1467-9280.1997.tb00427.x

12. Daniel J Simons and Christopher F Chabris. 1999. Gorillas in our midst: Sustained inattentional blindness for dynamic events. Perception 28 (1999), 1059-1074. DOI : http://dx.doi .org/10.1068/p281059

13. D. J. Simons, S. L. Franconeri, and R. L. Reimer. 2000. Change blindness in the absence of a visual disruption. Perception 29, 10 (2000), 1143-1154. DOI : http://dx.doi.org/10.1068/p3104

14. Daniel J. Simons and Daniel T. Levin. 1998. Failure to Detect Changes to People During a Real-World Interaction. Psychonomic Bulletin \& Review 5 (1998), 644. DOI : http://dx.doi.org/10.3758/BF03208840

15. Brian Cantwell Smith. 1996. On the origin of objects. MIT Press. I-XII, 1-420 pages.

16. Daniel Vogel and Ravin Balakrishnan. 2004. Interactive Public Ambient Displays: Transitioning from Implicit to Explicit, Public to Personal, Interaction with Multiple Users. In Proceedings of the 17th Annual ACM Symposium on User Interface Software and Technology (UIST '04). ACM, New York, NY, USA, 137-146. D0I : http://dx.doi.org/10.1145/1029632.1029656 